\title{
Similarities and Differences Among the Taiwan, China, and Hong-Kong Versions of the WHOQOL Questionnaire
}

\author{
Grace Yao $\cdot$ Chia-huei Wu
}

Accepted: 13 April 2008/Published online: 30 September 2008

(C) Springer Science+Business Media B.V. 2008

\begin{abstract}
To facilitate comparison across cultures, the World Health Organization (WHO) has been developing a universal measure of quality of life (QOL) called the WHOQOL Questionnaire. This questionnaire contains 24 facets organized into six broad domains: physical, psychological, level of independence, social relationships, environment, and spirituality/religion/personal beliefs. The standard WHOQOL is designed for cross-cultural comparison. However, to obtain a valid QOL measure that can be applied in diverse populations, cultural adaptation of the WHOQOL has been encouraged. Each culture is permitted to add culture-specific questions, called national items, so that the questionnaire can also reflect cultural attributes. Three Chinese versions of the WHOQOL have been developed for China, Hong Kong, and Taiwan. National items were selected for each version according to the criteria proposed by the WHOQOL Group. The purpose of this paper is to compare the Taiwan version to the China and Hong Kong versions. The questionnaire development process, response scale generation, psychometric properties (reliability and validity), national items, and population means were all examined. Results indicated that not only is cultural adaptation of WHOQOL measures necessary for individual cultures, but also for sub-cultures, as these differences must be considered in order to provide effective health care and treatment.
\end{abstract}

Keywords WHOQOL $\cdot$ Chinese versions $\cdot$ Culture

\section{Introduction}

Over the last 30 years, health care providers and researchers have agreed that treatment intervention should be evaluated in part by its impact on health-related quality of life (QOL) (Leung et al. 1997c). This perspective has motivated health care researchers to

G. Yao $(\bowtie) \cdot$ C.-h. Wu

Department of Psychology, National Taiwan University, No. 1, Sect. 4, Roosevelt Rd, 10617 Taipei,

Taiwan, China

e-mail: kaiping@ntu.edu.tw 
search for better QOL measures to assess treatment and evaluate cost-effectiveness. A number of QOL measures have been published, such as the Sickness Impact Profile (SIP), the Nottingham Health Profile (NHP), and the Short-Form-36 Health Survey (MOS SF-36). These instruments have been translated into different languages for cross-cultural or crossnational administration. As a result, the issue of cross-cultural or cross-national validation and equivalence of QOL measurement has become an important part of measure development.

Herdman et al. (1997) reviewed 66 recently published articles regarding health-related QOL measures that had been adapted for other countries and found 19 different types of equivalence. The most frequently mentioned types of equivalence included conceptual, semantic, functional, scale/metric, technical, and operational. They found that different health-related QOL measures adopted different definitions of the same type of equivalence. In particular, there was substantial variation in how conceptual equivalence was defined. They concluded that the equivalence of these cross-culturally adapted QOL measures is not reliable.

Hui and Triandis (1985) postulated four levels of equivalence that an instrument must have in order to warrant comparison of data obtained in different cultures: conceptual/ functional equivalence, equivalence in construct operationalisation, item equivalence, and scalar equivalence. The most important type is conceptual equivalence. It refers to the meaningful communication and discussion of the concepts across cultures. Equivalence in construct operationalisation indicates the same transition process for a construct from theory to measurement across cultures. It implies a need to adopt the same questionnaire development procedure and administration mode cross-culturally. Item equivalence refers to use of the same items so that comparisons across cultures can be achieved. Lastly, scalar equivalence refers to use of the same metric in assessing a construct across cultures. It implies distributional identity across cultures.

To facilitate global comparisons of a variety of disease groups in a wide range of cultures, in 1991 the World Health Organization (WHO) initiated a cross-cultural project for the development of a QOL questionnaire (WHOQOL) for generic use. Field tests were completed in 1995 (Szabo 1996; The WHOQOL Group 1994a, b, 1995, 1998a; World Health Organization 1995a-d, 1996).

The WHOQOL project defined QOL as

\begin{abstract}
individuals' perceptions of their position in life in the context of the culture and value systems in which they live, and in relation to their goals, expectations, standards, and concerns. It is a broad-ranging concept, incorporating in a complex way the persons' physical health, psychological state, level of independence, social relationships, personal beliefs, and relationship to salient features of the environment. (Szabo 1996; The WHOQOL Group 1994b, 1995, 1998a, b; World Health Organization 1993, 1996)
\end{abstract}

By sampling approximately 5,000 people from 15 culturally diverse field centers, they developed the final WHOQOL-100 questionnaire (Szabo 1996; The WHOQOL Group 1994a, b, 1995, 1998a; World Health Organization 1995a-d, 1996). The WHOQOL-100 contains 24 facets organized into six broad domains: physical, psychological, level of independence, social relationships, environment, and spirituality/religion/personal beliefs. Each facet contains four items. Four additional items measure overall QOL and general health (forming Facet-G). The final version of the questionnaire contains 100 generic items and is designed for cross-cultural comparisons. However, each culture is allowed to add culture-specific questions, called national items, so that the questionnaire can also reflect 
particular cultural attributes (Sartorius and Kuyken 1994; World Health Organization 1994, 1995b). As of 2004, more than 40 language versions had been developed (Skevington et al. 2004b).

In past years, researchers have paid more attention to cross-cultural QOL comparisons (Power et al. 1999; Skevington et al. 2004a; The WHOQOL Group 1998a). Comparisons across sub-cultures are also of interest. For example, Liao et al. (2005) examined differences in subjective QOL between Taiwan and Hong Kong at both societal and individual levels. These two Chinese societies share a cultural heritage, but vary in their social and political systems. Although they found common factors contributing to individual QOL in both societies, they also found that factors associated with the economy play an important role in individual QOL in Hong Kong, while those associated with the whole society are more critical in Taiwan. This difference can be explained by the political and economic experiences of the two societies.

In this study, we analyze sub-culture differences in individual QOL among China, Hong Kong, and Taiwan by examining results from the three versions of the WHOQOL questionnaire created specifically for use in each of these regions. The documents detailing development of the three versions were mostly written in Chinese and never published. As a result, researchers in other cultures are unlikely to have access to these documents.

The purpose of this paper is to compare the Taiwan version to the China and Hong Kong versions in terms of the processes used to develop the version, generate the response scale, and select national items. The psychometric properties and population means of each of the three measures are also compared. Specifically, we used published research papers and unpublished reports from the WHOQOL Questionnaire Cultural Adaptation Group in China, (Fang 2000; Fang et al. 1998, 1999a, b), Hong Kong (Leung et al. 1997a-c; Leung and Lin 1997), and Taiwan (Lin et al. 1999; The WHOQOL-Taiwan Group 2000, 2005; Yao et al. 2002, 2007b). We expect to illustrate that the WHO should expand the purpose of cultural adaptation of the WHOQOL Questionnaire to include not only different cultures but also sub-cultures in order to maximize utility for social policies and program planning.

\section{Method}

In the following, the three Chinese versions are examined in terms of the questionnaire development process, response scale generation, psychometric properties (reliability and validity), content of national items, and the population means.

\subsection{The Development Process}

The WHOQOL group has published many documents to guide cultural adaptation of the questionnaire (Sartorius and Kuyken 1994; The WHOQOL Group 1994a, 1998a, b; World Health Organization 1992, 1993, 1994, 1995a-d, 1996), including the method to be used in questionnaire translation, the sampling plan, response scale generation, and national item generation. The WHOQOL Group recommends including both in-patients and outpatients as participants in the adaptation process, with approximately equal numbers of males and females above and below 45 years of age, with a ratio of 1:5 healthy to ill subjects (The WHOQOL Group 1998b; World Health Organization 1995b, 1996). China was the first of the three Chinese societies to participate in the WHOQOL project. They finished adapting the questionnaire in 1995 (Fang 2000; Fang et al. 1998, 1999). The Hong Kong version was finished in 1997 (Leung et al. 1997a-c; Leung and Lin 1997). Taiwan's version was 
completed in 1999 (Lin et al. 1999; The WHOQOL-Taiwan Group 2000, 2005; Yao et al. 2002).

The researchers in China used a two-stage random sampling plan from six large cities (Guangzhou, Beijing, Shanghai, Chengdu, Shenyang, and Xian) and selected 1,654 participants, of which 877 were patients (with cardiac disease, hypertension, osteoarthritis, cancer, diabetes mellitus, and bone fractures), and 777 were healthy participants. The Hong Kong researchers collected data from a total of 856 participants, of which most $(n=699)$ were sampled from 19 Hospital Authority institutes and six community rehabilitation organizations. Patient's problems included cardiac conditions, pulmonary conditions, neurological conditions, renal conditions, burns, psychiatric conditions, rheumatoid arthritis, and systemic lupus erythematosis, diabetes, cancer, chronic pain and patients whose limbs were amputated. For the Taiwan version, the whole of Taiwan was stratified into four regions. At least four hospitals were selected from each region including public and private medical centers and community hospitals. Each hospital contributed data from about 48 patients and 12 healthy volunteers. A total of 17 hospitals and 1,068 participants completed the questionnaire. Patients with a definite diagnosis of disease were classified as ill, while a patient's family members or volunteers without any symptoms were considered healthy. A total of 214 healthy and 854 ill participants, including 138 patients with diagnosis of cardiovascular disease, 39 with diabetes mellitus, 63 with cancer, 139 with gastrointestinal disorder, 83 with musculoskeletal disorder, and 87 with respiratory disorder, completed the questionnaire. Table 1 presents the demographic data for each subculture.

\subsection{Response Scale Generation}

According to Hui and Triandis (1985), a cross-cultural measure should demonstrate scalar equivalence with the original form. That is not to say that a culturally adapted measure should use only directly translated scale descriptors from the original measure, instead the measure should locate similar individuals at the same point on the scale across cultures. This requires investigation of equal-interval indigenous scale descriptors in each culture (Szabo et al. 1997) and is required by the WHO.

Each item in the WHOQOL questionnaire uses a five-point Likert-type response scale. There are four types of response scales: capacity, frequency, intensity, and evaluation. The WHOQOL Group claims that the anchor descriptors are universal, but requires researchers developing each language version to identify the three intermediate scale descriptors for each of the four types of scales by asking 20 lay persons to rate 15 or more potential scale descriptors on a $10 \mathrm{~cm}$ visual-analogue scale with two universal anchor descriptors (Szabo 1996; The WHOQOL Group 1994b; World Health Organization 1993). The best descriptors are selected according to the descriptive statistics (mean, median, standard

Table 1 The demographic data from the cultural adaptation of the WHOQOL questionnaire to three Chinese subcultures

\begin{tabular}{llll}
\hline & China & Hong Kong & Taiwan \\
\hline Gender ratio (M:F) & $838: 816$ & $425: 416$ & $530: 530$ \\
Health ratio (healthy:ill) & $777: 877$ & $157: 699$ & $214: 854$ \\
Age & $<45: 767, \geq 45: 887$ & $45.30 \pm 16.07$ & $41.57 \pm 14.83$ \\
\hline
\end{tabular}


Table 2 Response scale generation details

\begin{tabular}{|c|c|c|c|c|}
\hline & China & Hong Kong & & Taiwan \\
\hline \multicolumn{5}{|c|}{ Participants (20 suggested) } \\
\hline Gender (M:F) & $48: 42$ & $86: 79$ & $10: 20$ & $43: 110$ \\
\hline Age & $37: 45: 8^{\mathrm{a}}$ & $40 \pm 10.04$ & $32.47 \pm 10.04$ & $8: 52: 34: 17: 21^{b}$ \\
\hline Education & $18: 30: 42^{\mathrm{c}}$ & $32: 82: 47^{\mathrm{d}}$ & $3: 21: 6^{\mathrm{d}}$ & $10: 13: 53: 77^{\mathrm{e}}$ \\
\hline \multicolumn{5}{|c|}{ Potential scale descriptors (15+ suggested) } \\
\hline Capacity & 28 & 10 & 11 & 17 \\
\hline Frequency & 30 & 12 & 11 & 22 \\
\hline Intensity & 18 & 18 & 11 & 23 \\
\hline Evaluation & 22 & 27 & 11 & 18 \\
\hline
\end{tabular}

a Age (China): 18-29:30-49: $\geqq 50$

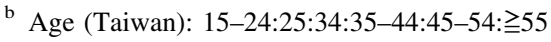

c Education (China):middle school:senior high school:college

d Education (Hong Kong):primary:high school:university and above

e Education (Taiwan):primary:middle school:high school:college and above

deviation, etc.). Taiwanese researchers followed the WHOQOL recommendation, but used more participants and more potential response scale descriptors. Table 2 provides information about the process for selecting the response scale descriptors for each of the three Chinese language versions of the WHOQOL. Hong Kong researchers conducted the descriptor selection process twice to improve the $25 \%$ and $75 \%$ descriptors. Table 3 presents the actual response scale descriptors selected for each of the three versions with the original Chinese and English back-translation terms as well as the standard English terms (Fang 2000; Fang et al. 1999b; Leung and Lin 1997; Lin et al. 1999).

\subsection{Psychometric Properties}

Several types of standard psychometric properties (reliability and validity indices) were compared among the three Chinese versions. For example, the internal consistency (Cronbach's $\alpha$ ) coefficients were calculated at facet and domain levels. A Cronbach's $\alpha$ coefficient above 0.70 is acceptable. For the Hong Kong and Taiwan versions, the testretest reliability (intra-class) coefficients with an interval less than 1 month were calculated at item, facet, and domain levels.

Content validity and concurrent validity were evaluated by calculating Pearson's correlation coefficients between variables, namely, between each item and its hypothesized facet/domain, between each facet and its hypothesized domain, among all domains, and between each item/domain and items G1, G4, and Facet-G. Item G1 and G4 are the two items from Facet-G measuring overall QOL and general health respectively. The correlation between each domain and a five-point Likert-type scale item (from very bad to very good) measuring current health status was also calculated. An item with good content validity should have a higher correlation with its hypothesized facet/domain than with other facets/domains. A high correlation among items/domains and G1/G4/Facet-G and current health status indicates that the items and domains have good concurrent validity.

A multiple regression analysis was conducted to evaluate predictive validity using the six domain scores as the predictor variables and the Facet-G score as the criterion variable. A high R-square (\% score variance explained) indicates good predictive validity. 


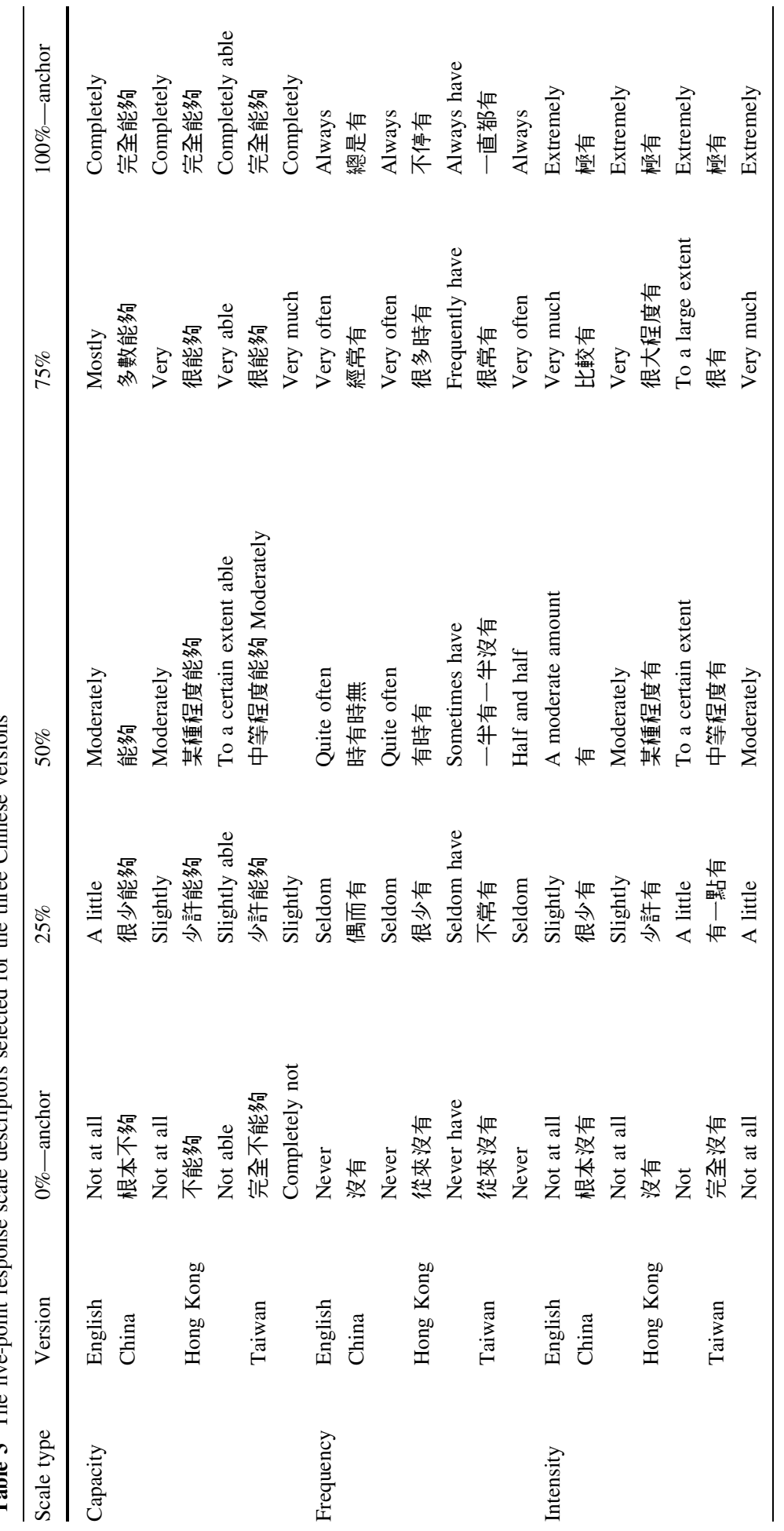




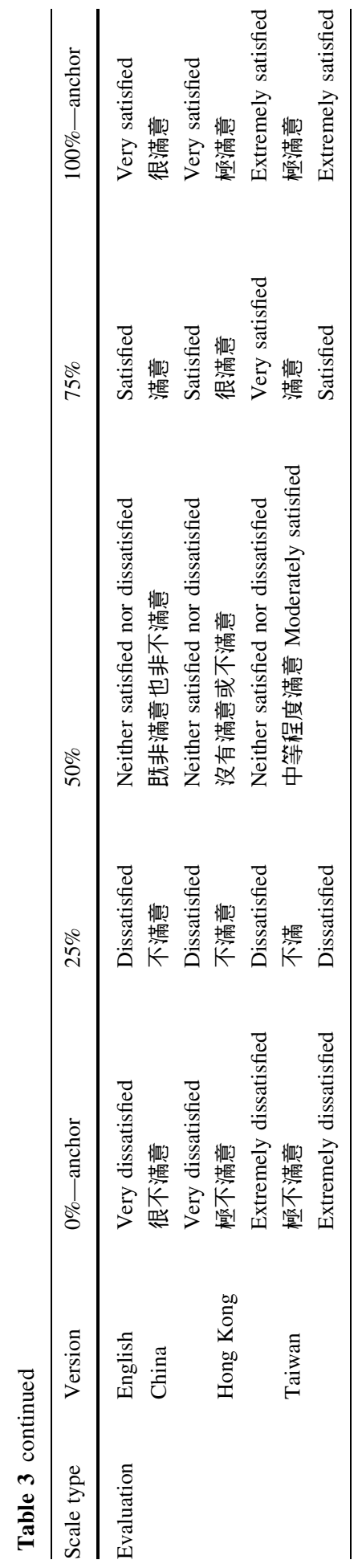


Discriminant validity was tested with independent sample $t$-tests on the results from healthy and ill participants on each item, facet, and domain. To examine construct validity, both exploratory factor analysis (EFA) and confirmatory factor analysis (CFA) were conducted. EFA using the iterative principal factoring axis method with promax rotation was applied. CFA was also conducted to test construct validity on the original six-factor model (physical, psychological, independence, social relationships, environment, and spirituality/religion/personal beliefs) and a simplified four-factor model (physical combined with independence, psychological combined with spiritual). Table 4 illustrates the psychometric properties of the three Chinese versions.

\subsection{National Item Selection}

The WHOQOL Group encourages each culture to select national items reflecting cultural aspects. Researchers developing each of the Chinese-language versions followed the suggestions of the WHOQOL Group to select their own national items (World Health Organization 1992) (e.g., conducting focus groups to propose potential items; conducting item analysis to select better items). Selection of national items is supposed to be based on psychometric criteria (Leung et al. 1997; The WHOQOL Group 1998a, b; World Health Organization 1996). That is, the psychometric properties of the national items should be at least as good as existing items in the same domain and facet. Specifically, a better item should explain a greater amount of the total variance of the WHOQOL and Facet-G, and the variance of its hypothesized facet/domain. The item should also distinguish between the QOL of healthy and ill participants. A model with the selected items should show good factor structure. Table 5 illustrates the national items selected for the three Chinese versions with the corresponding English back-translation. Researchers developing the China version selected three items; 12 items were selected for the Hong Kong version; and Taiwan researchers also selected 12 items for their version. Similar items among the three Chinese versions are highlighted.

\subsection{Population Means}

Following the recommendation of the WHOQOL (The WHOQOL Group 1998b; World Health Organization 1995b, 1996) both healthy and ill participants were included in the process of cultural adaptation. Table 6 lists the average and standard deviation of the six QOL domains for the healthy and ill samples for each Chinese version. Population means can be compared among the three versions and across the six QOL domains for both healthy and ill samples. Analysis of variance (ANOVA) tests with Tukey post-hoc paired group comparisons were conducted to compare the scores of the three versions. Since the sample for each area cannot fully represent the whole population for each area, the group differences among the three regions are presented only as a reference; they are not definitive.

\section{Results}

\subsection{Comments on the Development Process}

Fewer published and unpublished documents were available to represent the data from China than from the other two areas. Researchers in all three areas selected both healthy 


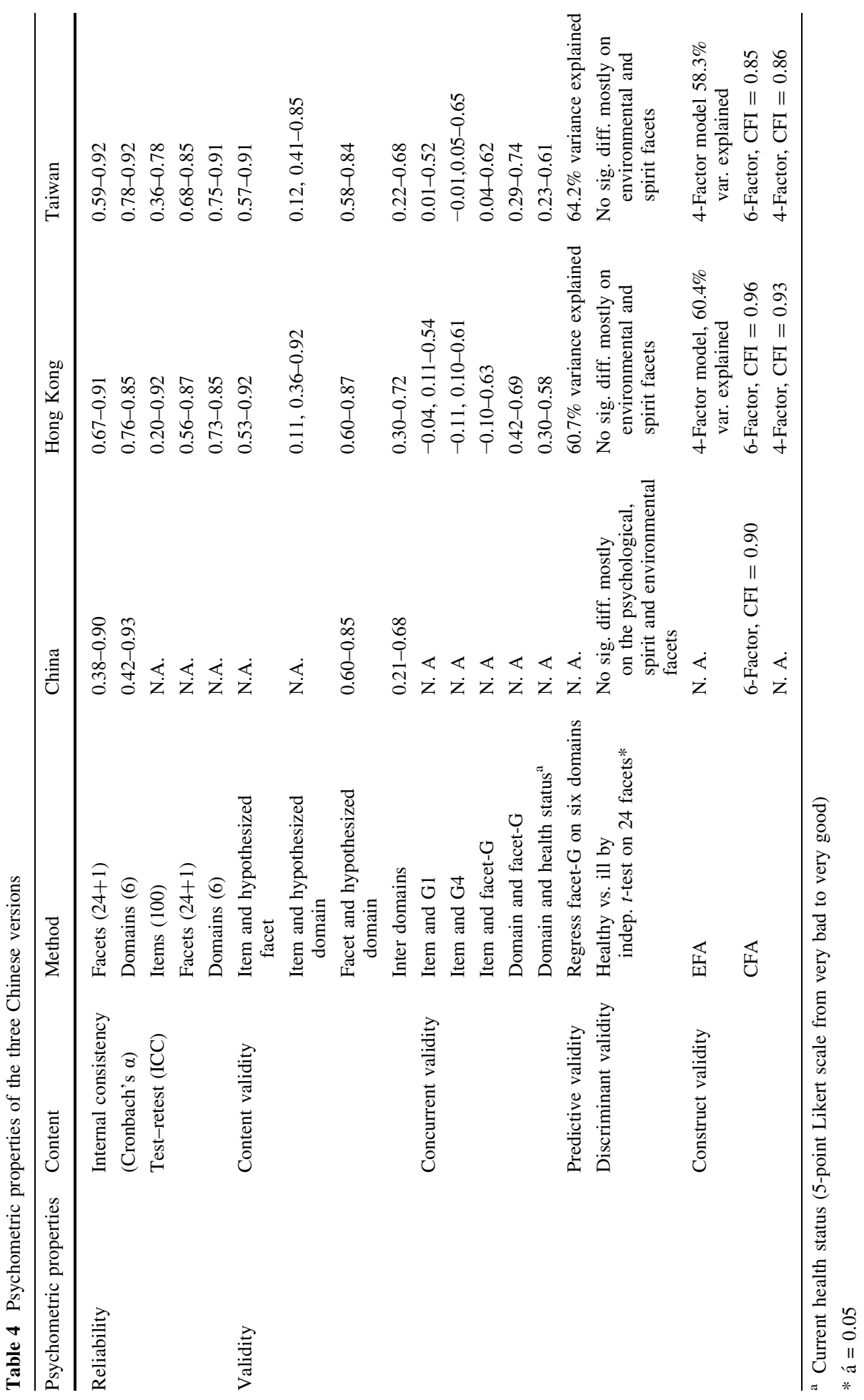




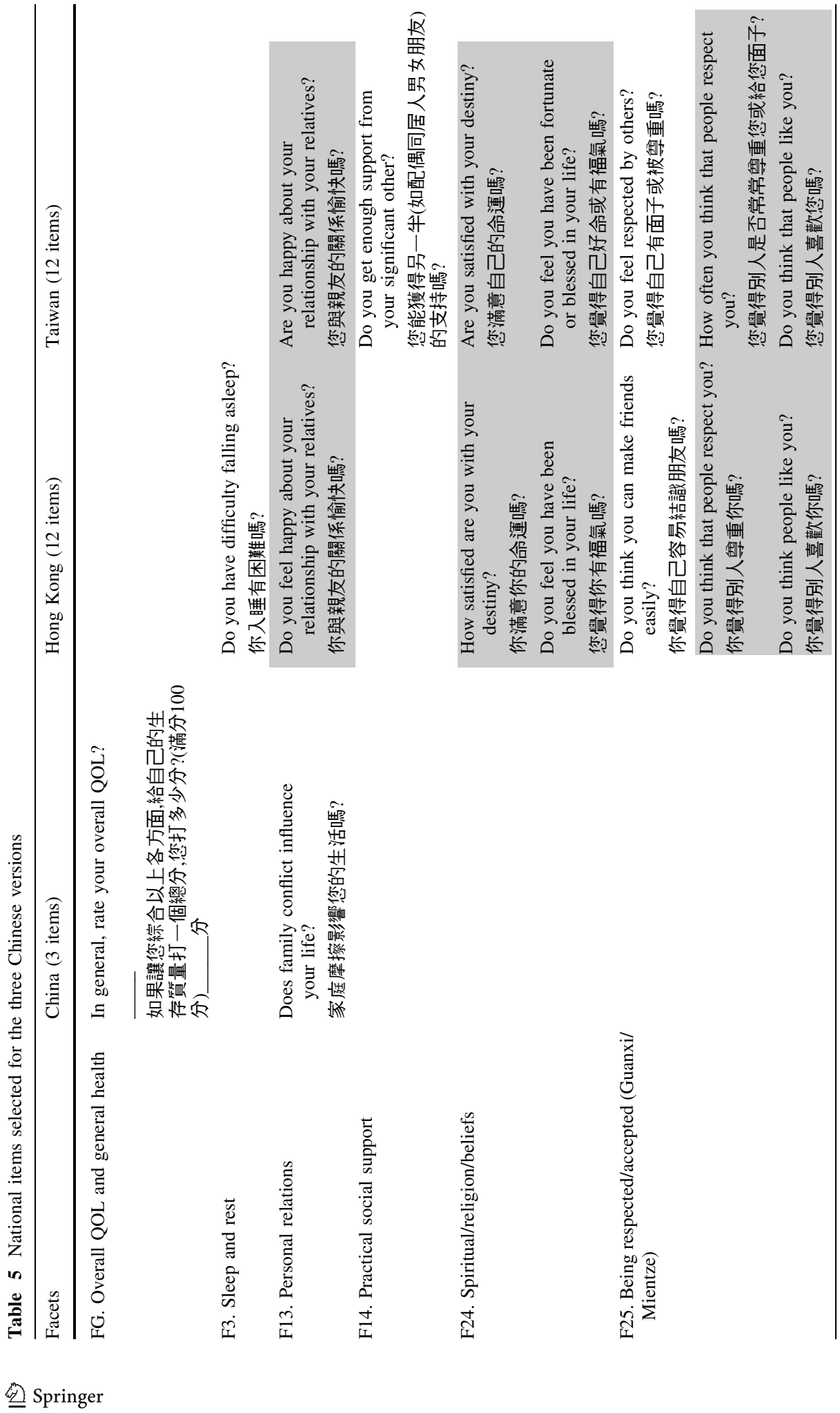




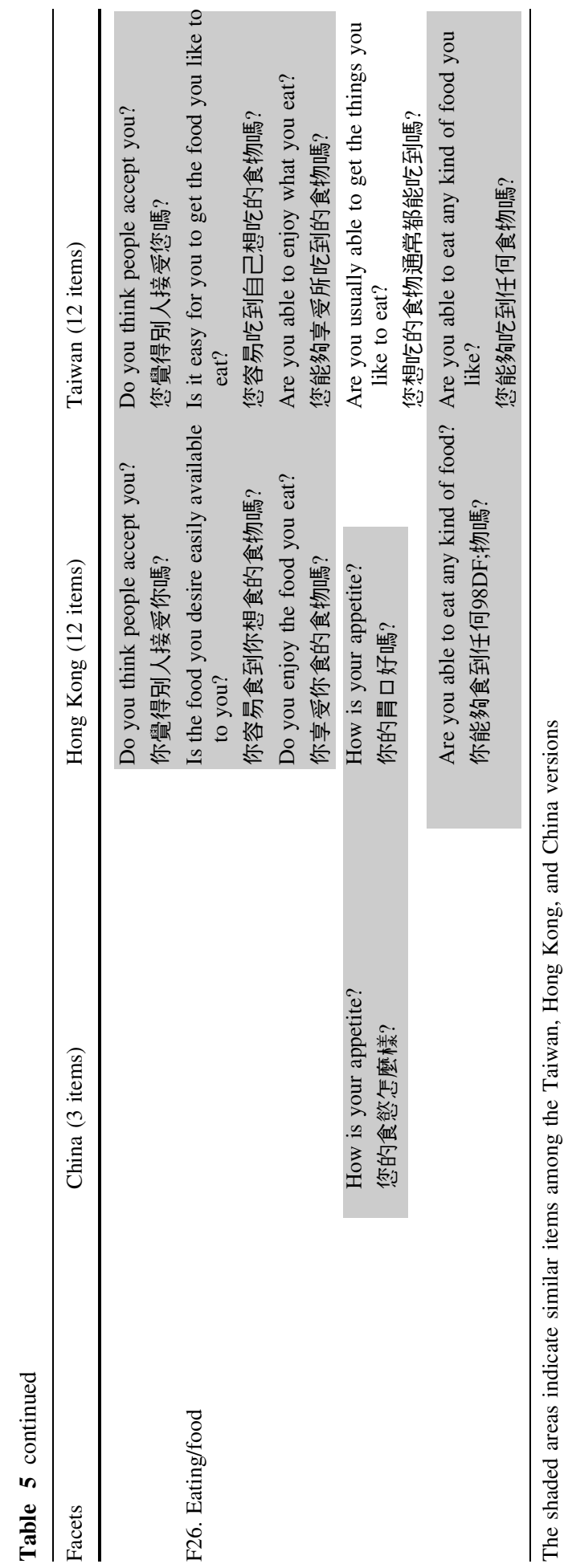


Table 6 The average and standard deviation of the six QOL domains for healthy and ill participants in the three Chinese versions

\begin{tabular}{llllll}
\hline Healthy sample & China & Hong Kong & Taiwan & F (2, 1145) & Post-hoc \\
\hline Physical & $15.10 \pm 2.30$ & $15.22 \pm 2.48$ & $15.28 \pm 1.94$ & 0.62 & \\
Psychological & $13.89 \pm 1.89$ & $14.40 \pm 2.31$ & $14.09 \pm 1.93$ & $4.72^{* *}$ & $\mathrm{H}>\mathrm{C}$ \\
Independence & $15.64 \pm 2.22$ & $17.22 \pm 1.86$ & $17.01 \pm 1.68$ & $62.10^{* *}$ & $\mathrm{H}>\mathrm{C}, \mathrm{T}>\mathrm{C}$ \\
Social & $13.93 \pm 2.06$ & $14.51 \pm 2.27$ & $14.60 \pm 1.89$ & $11.90^{* *}$ & $\mathrm{~T}>\mathrm{C}, \mathrm{H}>\mathrm{C}$ \\
Environmental & $12.14 \pm 2.08$ & $13.95 \pm 2.17$ & $13.38 \pm 2.00$ & $67.20^{* *}$ & $\mathrm{H}>\mathrm{T}, \mathrm{H}>\mathrm{C}, \mathrm{T}>\mathrm{C}$ \\
Spirit & $11.05 \pm 3.68$ & $15.10 \pm 3.42$ & $13.08 \pm 3.40$ & $95.20^{* *}$ & $\mathrm{H}>\mathrm{T}, \mathrm{H}>\mathrm{C}, \mathrm{T}>\mathrm{C}$ \\
\hline Ill sample & China & Hong Kong & Taiwan & $\mathrm{F}(2,2427)$ & Post-hoc \\
\hline Physical & $14.10 \pm 2.62$ & $14.02 \pm 2.95$ & $13.71 \pm 2.46$ & $5.07^{* *}$ & $\mathrm{C}>\mathrm{T}$ \\
Psychological & $13.89 \pm 1.92$ & $13.77 \pm 2.51$ & $13.46 \pm 1.96$ & $12.40^{* *}$ & $\mathrm{C}>\mathrm{T}, \mathrm{H}>\mathrm{T}$ \\
Independence & $14.69 \pm 2.53$ & $13.79 \pm 3.10$ & $15.16 \pm 2.57$ & $49.60^{* *}$ & $\mathrm{~T}>\mathrm{C}, \mathrm{T}>\mathrm{H}, \mathrm{C}>\mathrm{H}$ \\
Social & $14.18 \pm 2.00$ & $13.68 \pm 2.57$ & $13.95 \pm 2.04$ & $10.10^{* *}$ & $\mathrm{C}>\mathrm{T}, \mathrm{C}>\mathrm{H}$ \\
Environmental & $12.51 \pm 2.28$ & $13.86 \pm 2.32$ & $12.91 \pm 1.86$ & $78.70^{* *}$ & $\mathrm{H}>\mathrm{T}, \mathrm{H}>\mathrm{C}, \mathrm{T}>\mathrm{C}$ \\
Spirit & $11.05 \pm 3.48$ & $12.85 \pm 4.25$ & $12.80 \pm 3.07$ & $68.40^{* *}$ & $\mathrm{H}>\mathrm{C}, \mathrm{T}>\mathrm{C}$ \\
\hline
\end{tabular}

The critical value for $F(0.05 ; 2,1145)$ is 3.00 and for $F(0.01 ; 2,1145)$ is 4.62

The critical values for $F(0.05 ; 2,2427)$ is 3.00 and for $F(0.01 ; 2,2427)$ is 4.61

$* * p<0.01$

and ill samples, and used approximately equal numbers of males and females. Both Hong Kong and Taiwan researchers followed the WHOQOL recommendation (The WHOQOL Group 1998b; World Health Organization 1995b, 1996) to collect more ill than healthy participants, but researchers in China used a relatively equal number of healthy and ill participants. The composition of the ill sample differed among the three versions. The average age was a little younger in the Taiwan sample.

\subsection{Comments on Response Scale Generation}

Researchers in all three areas used more participants and more potential scale descriptors to develop the response scale descriptors than required by the WHOQOL Group (Szabo 1996; The WHOQOL Group 1994a, b, 1995; World Health Organization 1993, 1995b). Although the WHOQOL Group has claimed that the anchor descriptors are universal, both Hong Kong and Taiwan researchers used stronger anchor descriptors (i.e., extremely dissatisfied/ satisfied) on the evaluation scale, according to the English back-translation. The Hong Kong version contains more vague terms for the middle descriptors on each of the four types of scales (e.g., to a certain extent, sometimes), while the Taiwan version has very clear middle terms (e.g., half and half, moderately). The $25 \%$ and $75 \%$ descriptors were similar in the three versions. Readers interested in response scale generation can refer to the original references for detailed empirical information (Fang et al. 1998; Leung and Lin 1997; Lin et al. 1999; The WHOQOL-Taiwan Group 2005).

\subsection{Comments on the Psychometric Properties}

The documentation for the Hong Kong and Taiwan versions was more complete than for the China version. For example, test-retest reliability, concurrent validity, prediction 
validity, and construct validity by EFA were missing for the China version. Researchers from all three areas claimed that their versions were reliable and valid. However, the Hong Kong and Taiwan versions had the most similar psychological properties.

\subsection{Comments on the National Items Selection}

National items were selected according to the psychometric criteria proposed by the WHOQOL Group (Leung et al. 1997; The WHOQOL Group 1998a, b; World Health Organization 1996). The same two new facets ("being respected/accepted" and "eating/ food") were proposed by both Hong Kong and Taiwan researchers. However, according to the advanced psychometric analyses, the Taiwan version classified these facets into the social relationships and environment domains respectively, while Hong Kong researchers classified both facets into the psychological domain (The WHOQOL-Taiwan Group 2005; Yao et al. 2002). As Table 5 shows, both the Taiwan and Hong Kong researchers selected 12 national items while China researchers selected only three national items. Taiwan researchers proposed national items for the personal relationship, practical social support, spirituality/religion/beliefs, being respected/accepted (guanxi/mianzi, 關係/面子), and eating/food facets. Hong Kong researchers selected similar national items for the same facets, although they excluded the practical social support facet and added a sleep and rest facet. One of the items in the China version was an overall rating of QOL, and the other two were placed under the personal relationship and eating/food facets. Nine out of 12 items in the Taiwan and Hong Kong versions were fairly similar (see the highlighted areas in Table 5). However, only one item in the China version was similar to the Hong Kong version. Readers interested in national item selection can refer to the original references for detailed empirical information (Fang et al. 1998, 1999a; Leung et al. 1997; The WHOQOL-Taiwan Group 2005; Yao et al. 2007a).

\subsection{Comments on Population Means}

According to Table 6, the population means for the healthy samples were significantly different among the three versions in the psychological, independence, social, environmental, and spiritual domains. The exception was the physical domain. Generally, the China sample scored lowest in all domains, while the Hong Kong sample had higher scores, and the Taiwan sample was in the middle. Similarly, population means for the ill samples were significantly different in all six domains. The China sample generally had higher scores than the Hong Kong and Taiwan samples.

\section{Discussion and Conclusion}

\subsection{The Development of the Three Chinese Versions}

The original purpose in developing the WHOQOL questionnaire was to facilitate global comparisons on a variety of disease groups in a wide range of cultures. To culturally adapt this questionnaire, the WHOQOL Group recommended a ratio of 1:5 healthy to ill participants. Except for the smaller sample of ill participants in the China version, the three Chinese versions were basically developed according to the WHOQOL Group's recommendations for cultural adaptation. 
For the five-point response scale generation, the $25 \%$ and $75 \%$ descriptors were similar among the three Chinese versions. However, the middle descriptors and two extreme descriptors on the evaluation scale were different according to the English back-translations. This difference indicates not only that the effect of sub-cultures should be considered, but also that the assumption of universal anchor points proposed by the WHOQOL Group may need to be challenged. Further studies should be conducted to clarify the issue.

All three Chinese versions were shown to be reliable and valid. However, the Hong Kong and Taiwan versions were more similar in psychometric properties and national item selection. This may imply that Hong Kong and Taiwan share more similar attributes. Nevertheless, the two newly proposed facets ("being respected/accepted" and "eating/ food") were classified into different domains in each version, suggesting that the impact of subcultures cannot be ignored.

Hagerty et al. (2001)pointed out that a generic QOL instrument must have relevance for most people. QOL measures designed with a specific target population in a specific social context may not capture the totality of life experiences for other populations in different contexts and settings. Since there is a greater under-representation of healthy participants than participants with illness, the applicability of the WHOQOL questionnaire to the general population is questionable. In our recent study (Yao et al. 2007a), we compared psychometric properties by using the sample in this paper $(n=1,068)$ and a randomly selected sample from Taiwan's 2001 National Health Interview Survey (NHIS) $(n=13,010)$. The ratio of healthy to ill participants was 1:4 for this paper and 2.33:1 in the 2001 NHIS study. The results showed that both samples had very similar psychometric properties. We concluded that the WHOQOL questionnaire can be applied to both healthy and ill samples in Taiwan.

\subsection{National Items Selection for the Three Chinese Versions}

\subsubsection{Similarities}

Similarities among the three Chinese sub-cultures are found in language, attitudes toward eating and food, the notion of fate, and the influence of Confucianism. People from the three regions basically share the same written language system, although the characters are written differently (simplified vs. traditional) so that people in one region may not feel comfortable or competent in reading the characters common in a different region. All three areas use the same official spoken language (Mandarin), however, in Hong Kong the most widely used dialect is Cantonese, which is not understandable to people fluent only in Mandarin. A greater proportion of the elderly Taiwanese population speaks only Hokkien. There is also a diversity of local dialects in China that are not understandable to people who speak only Mandarin.

Another trait shared by the three sub-cultures is the importance of eating and food. In all three areas it is common for people to greet one another by asking "Are you full?" or "Have you eaten your meal yet?" Being unable to eat what one desires because of an illness is considered serious. Moreover, in Chinese, the words for good appetite (好食慾, 好胃口) literally mean good health. Although all three versions included items related to eating, this does not mean that the emphasis on eating is unique to Chinese. Instead, it indicates that the standard items on the WHOQOL questionnaire do not contain some items that are important to Chinese cultures. Skevington et al. (1999) pointed out that the issue of eating was considered during the qualitative stage of the WHOQOL Group's conceptual 
development work. However, it was taken out because of the limited number of facets for the final version of the WHOQOL. The emphasis on eating should be further studied in order to determine whether it is found in other cultures; eating items may be designed in different ways in different cultures.

Both Taiwanese and people from Hong Kong focus on destiny. They attribute their destiny to unknown gods, fatalistically and positively accepting what the gods give them. They also consult fortune tellers in predicting the future. A good destiny and many blessings from the gods may mean prosperity, many filial descendants, and that their desires will be fulfilled. People experience a favorable QOL if they are satisfied with their destiny and feel that they can die without regret.

The principles of ancient Confucianism still deeply influence Chinese thought, attitude, and behavior in modern Chinese societies. Confucian beliefs underlie personal relationships. All three versions proposed items for the personal relationships facet. The concept of personal relationships for culturally Chinese people differs from that of westerners. Western culture stresses an independent, distinct, and unique self (individualism) while Chinese culture, like many non-Western cultures, addresses an interdependent self and connection with others (collectivism) (e.g., Markus and Kitayama 1991, 1994; Triandis 1994). As a result, self-image, self-esteem, self-independence, and self-achievement are very important concepts for the Westerner. Westerners feel more satisfied with their lives if they have a higher self-esteem and self-independence whereas culturally Chinese people place a high value on having a web of personal connections with others and interdependence of the self. In personal relationships, one's role is not defined by one's unique character but by one's social relationships with others. In other words, harmonious relationships are very important in Chinese societies. All versions proposed items relating to maintaining harmonious relationships not only with their immediate family but also with their relatives. In the Confucian system, harmonious relationships are necessary in order to obtain a balanced interpersonal relationship between oneself and others. They are not required for the purpose of satisfaction or support from the relationship (Ho 1993). Markus and Kitayama $(1991,1994)$ believe that both the concepts of independent-self and interdependent-self exist in all cultures, however, different cultures may emphasize different parts of the concepts in people's beliefs, motivations, emotions, cognition, behaviors, and perceptions, so people are shaped differentially. All three Chinese versions of the WHOQOL have taken these factors into account.

Both the Taiwan and Hong Kong versions proposed items on the being respected/ accepted (guanxi/mianzi, 關係/面子) facet. In Chinese societies, being respected or accepted means that one has good connections (guanxi) with others so that others are more willing to do one a favor, returning or giving face (mianzi). Applying Western social exchange theories, Hwang $(1987,1995)$ studied Chinese relationalism and proposed a model of face and favor. He later analyzed the structure of Confucian morality and put forward a paradigm for Chinese conflict resolution on the basis of this model (Hwang 1995, 1997/1998). Ren (benevolence, 仁), yi (justice, 義), and li (courtesy, 禮) are at the center of Confucian morality. These three principles constrain Chinese social relationships. In Hwang's model, ren is an awareness of others and the environment. It is the principle of judging interpersonal relationships (guanxi; 關係) according to the degree of one's closeness with others. Three types of interpersonal relationships are specified in Hwang's model: instrumental ties, mixed ties, and expressive ties.

$Y i$ indicates that one should choose the appropriate social exchange rule for distributive justice according to the type of interpersonal tie with others. Three social exchange rules are noted: the equity rule, the equality rule, and the need rule. The rule to be applied is 
decided by the degree of closeness, or the type of relationship one has with the other person. $Y i$ entails doing what is right according to the proper social exchange rule. The three types of interpersonal ties respectively correspond to the three social exchange rules.

$\mathrm{Li}$ indicates that one must be courteous towards others no matter what decision one makes in the prior judgments. In Hwang's guanxi model, culturally Chinese people always first consider the degree of closeness between the self and others and then decide on the appropriate action. In the Taiwan version, this belief is reflected in the proposed national item about getting support from significant others.

\subsubsection{Dissimilarities}

The differences in selection of national items in the three Chinese versions of the WHOQOL questionnaire reflect each sub-culture's historical background, geographical position, and degree of influence from outside cultures. Unlike the Taiwan and China versions, the Hong Kong version proposed this item for the sleep and rest facet: "Do you have difficulty falling asleep?" As people in Hong Kong lead fast-paced lives and encounter a lot of pressure from their work, falling asleep was decidedly a prominent concern for Hong Kong respondents.

The Taiwan and Hong Kong versions shared many similar facets and items within facets that the China version did not have. This difference can be attributed to a combination of historical and geo-political factors. Both Hong Kong and Taiwan are located on the southeast coast of China and are comprised of several islands with some larger islands. However, part of Hong Kong's territory connects to China's Canton Province while Taiwan is geographically separated from China. Taiwan and Hong Kong were a part of China several hundred years ago. Taiwan has been occupied by a number of different foreign powers, such as the Dutch (1624-1661), the Spanish (1626-1642), and most recently the Japanese (1895-1945). At the end of World War II, Taiwan came under the administrative control of the Chinese Nationalist Party (Kuomintang, KMT). Taiwan's population is composed of aborigines $(<2 \%)$, Fukienese and Hakka $(85 \%$, the early immigrants from southern China), and later immigrants $(<15 \%)$ from China after World War II, mostly in 1949 after the defeat of the Nationalists. The majority of the population in Hong Kong are Chinese (96\%, mostly Cantonese), with Westerners, Malaysians, and Indians in the minority (4\%). Their different historical backgrounds have allowed them to develop diverse sub-cultures. More direct contact with nonChinese cultures has allowed faster access to modernization, industrialization, and westernization in Hong Kong and Taiwan in comparison to China. Of the three regions, Hong Kong seems to be most influenced by western cultures because of its former ties with Britain. As a result, a more Western-style individualism is more likely to be found in Hong Kong relative to Taiwanese and Chinese societies. Taiwanese have been heavily influenced by Japan due to the 50 years of colonization. As a result, the two new proposed facets were placed in the psychological domain in the Hong Kong version but not in the Taiwan version.

China has been governed by the Communists since 1949 and has been relatively more conservative in terms of accepting influence from outside cultures. However, in the last several years, China has begun opening up. We believe that the more China opens up, the more other cultures will influence traditional Chinese society as in Hong Kong and Taiwan. However, we also believe that Confucian beliefs will continue to play an important role in all of these culturally Chinese societies. 


\subsection{The Significance of Subcultures}

Questionnaires are a very common measurement tool in the social and behavioral sciences. Past research has shown that people in different cultures have different perceptions of the scale descriptors and the traits measured by a questionnaire (Forgas et al. 1977; Hermann and Raybeck 1981; Rudmin 1994; Taylor et al. 1972; Tzeng and Everett 1985; Tzeng et al. 1976; Waston et al. 1998). To assure cross-cultural adaptation of the WHOQOL questionnaire, the WHOQOL Group has published many guidelines (World Health Organization 1992, 1993, 1994, 1995a-d, 1996). This action allows the equivalence of WHOQOL questionnaires across countries so that global comparisons become possible.

In past literature, many studies have compared the similarities and differences across cultures in diverse research fields as well as in QOL research (Oishi et al. 1999; Skevington et al. 2004; Ware et al. 1998). However, Skevington and Tucker (1999) pointed out that "not only may concepts of QOL differ between countries, but also there may be many diverse cultural and linguistic groups and therefore meanings, within a single nationality (p. 51)". This observation supports the necessity of cross-cultural as well as cross-subcultural adaptation of the questionnaire. In the past, a few studies demonstrated similarities and differences across diverse subcultures (King and Bu 2005; Lee 1989; Sprecher and Toro-Morn 2002; Wang 2002; Xia and Qian 2001; Zhang 1997). For those studies, the class of subcultures as well as cultures was usually treated as a demographic variable and the purpose of those studies was to find the differences in dependent variables (such as mental health, leisure participation, etc.) in different subcultures.

In the current study, however, we quantitatively and qualitatively compared the WHOQOL questionnaire in three Chinese subcultures. For example, we compared the response scale descriptors and the national items selected by the three Chinese subcultures and found similarities as well as dissimilarities. Although the people in the three regions use a similar written language, now have the same official spoken language, and are deeply influenced by the same ancient Chinese philosophies, variations were still found in the three versions of the WHOQOL. Differences in historical experience led researchers to develop different versions of the QOL measure. This difference can also been found in other Chinese versions of QOL health surveys (e.g., SF-36 has China, Hong Kong, and Taiwan versions) as well as in several English versions of QOL measures (e.g., SF-36 and the WHOQOL have USA, Canadian, UK, and Australian versions) (Fuh et al. 2000). This study supports the claim that different versions of the WHOQOL questionnaire are needed for measuring peoples' QOL in different cultures and subcultures. In addition, as mentioned in Shih's paper, Chinese traditional philosophies such as the theory of yin and yang, and the five phases as well as Confucianism, Taoism, and Buddhism not only influence Chinese patients' values and beliefs, but also determine their perceptions of health, illness, and nursing care (Shih 1996). Health professionals should be more sensitive not only to patients' cultural background but also to sub-cultural backgrounds in order to provide the most effective treatment and health care.

In summary, in the past, many studies have compared similarities and differences across cultures. However, our study showed that differences across subcultures exist as well. To date, no studies have addressed differences in subcultures or the need for development of additional subcultural measures. The approach of this study provides a new avenue for cross-cultural and cross-subcultural research. In other words, when cultures and subcultures are compared, the measurement instrument can be a media. Different cultural and subcultural values may be reflected in questionnaire development via item selection and response scale generation. The implication of this study is that similarities and 
dissimilarities can be found not only within cultures but also within subcultures. Investigating the similarities and dissimilarities within cultures and subcultures can also be achieved through comparisons of questionnaire development, especially item selection and response scale generation.

\subsection{Limitations of the Study}

There are several limitations to this study. First, the results of this study are based on published and unpublished documents from the WHOQOL Questionnaire Cultural Adaptation Group from each of the three societies. We did not have access to the original data. As a result, the China version had quite a lot more missing information. Second, although this study attempted to compare the three Chinese versions, the different population compositions may have hindered the analysis. For example, more healthy participants were selected for the China version, the types of ill participants were different among the three versions; and participants in the development of the China version were recruited only from six large cities, while those for the Hong Kong and Taiwan versions were recruited from more varied areas. These differences may result in difficulty interpreting the mean differences on the WHOQOL. However, a recent study showed that the WHOQOL can be used for healthy people as well as ill ones with pretty similar psychometric properties and factor structure (Yao et al. 2007a; Yao and $\mathrm{Wu} 2005$ ). It is reasonable to assume that the comparison among the three Chinese versions was meaningful. Third, this study borrowed cross-cultural theories to discuss the similarities and dissimilarities of the three Chinese versions. However, the appropriateness of applying these theories to subcultures needs to be further investigated.

Acknowledgement The study was supported by grants from the National Science Council. (NSC 87-2312B-002-001, NSC 88-2314-B-002-344, NSC 89-2312-B-002-001, NSC 95-2413-H-002-002, NSC 96-2413H-002-001-MY3). Special thanks to Dr. Fang Jiqian and Mr. Leung Kwok Fai for providing the WHOQOL research papers and documents from the China and Hong Kong versions respectively.

\section{References}

Fang, J. Q. (2000). Quality of life: Evaluation methods and applications (pp. 113-143). Beijing: Peiking University Medical Press (in Chinese).

Fang, J. Q., Hao, Y. T., \& Li, C. (1999a). Reliability and validity for the Chinese version of WHO quality of life scale. Chinese Mental Health Journal, 13, 203-205 (in Chinese).

Fang, J. Q., Shi, M. L., Zhou, F. Q., \& Hao, Y. T. (1999b). Cultural adaptation of WHOQOL-100 from English to Chinese. Unpublished report. Guangzhou, China: Sun Yat-Sen University of Medical Sciences.

Fang, J. Q., Li, C. X., Hao, Y. T., et al. (1998). The development of the WHOQOL-100 China version. Statistics and Prediction, suppl., 40-41 (in Chinese).

Forgas, J. P., Kagan, C., \& Frey, D. (1977). The cognitive representation of political personalities: A crosscultural comparison. International Journal of Psychology, 12, 19-30.

Fuh, J. L., Wang, S. J., Lu, S. R., Juang, K. D., \& Lee, S. J. (2000). Psychometric evaluation of a Chinese (Taiwanese) version of the SF-36 health survey amongst middle-aged women from a rural community. Quality of Life Research, 9, 675-683.

Hagerty, M. R., Cummins, R. A., Ferriss, A. L., Land, K., Michalos, A. C., Peterson, M., et al. (2001). Quality of life indexes for national policy: Review and agenda for research. Social Indicators Research, $55,1-91$.

Herdman, M., Fox-Rushby, J., \& Badia, X. (1997). 'Equivalence' and the translation and adaptation of health-related quality of life questionnaires. Quality of Life Research, 6, 237-247.

Hermann, D. J., \& Raybeck, D. (1981). Similarities and differences in meaning in six cultures. Journal of Cross-Cultural Psychology, 12, 194-206. 
Ho, D. Y. F. (1993). Relational orientation in Asian social psychology. In U. Kim \& J. W. Berry (Eds.), Indigenous psychologies: Research and experience in cultural context (pp. 240-259). Newbury Park, CA: Sage.

Hui, C. H., \& Triandis, H. C. (1985). Measurement in cross-cultural psychology: A review and comparison of strategies. Journal of Cross-Cultural Psychology, 16, 131-152.

Hwang, K. K. (1987). Face and favor: The Chinese power game. American Journal of Sociology, 92, 944-974.

Hwang, K. K. (1995). Knowledge and action: The socio-psychological explanation on traditional Chinese Culture. Taipei: Psychology Publishing (in Chinese).

Hwang, K. K. (1997/1998). Guanxi and Mientze: Conflict resolution in Chinese Society. Intercultural Communication Studies, 7, 17-37.

King, R. C., \& Bu, N. (2005). Perceptions of the mutual obligations between employees and employers: A comparative study of new generation IT professionals in China and the United States. International Journal of Human Resource Management, 16, 46-64.

Lee, E. (1989). Assessment and treatment of Chinese-American immigrant families. Journal of Psychotherapy \& the Family, 6, 99-122.

Leung, K. F., Chan, C. C. H., \& Lin, F. (1997a). Cultural adaptation of the WHOQOL-100 and the development of national questions for the Hong Kong Chinese version WHOQOL. Unpublished report. Hong Kong: Hong Kong Hospital Authority.

Leung, K. F., Chu, M. M. L. \& Lau, J. T. F. (1997b). Cultural relevancy of WHOQOL structure in Hong Kong Chinese. Unpublished report. Hong Kong: Hong Kong Hospital Authority.

Leung, K. F., \& Lin, F. (1997). Development of Hong Kong chinese response scales for the WHOQOL (HK). Unpublished report. Hong Kong: Hong Kong Hospital Authority.

Leung, K. F., Tay, M., Cheng, S. W., \& Lin, F. (1997c). Hong Kong Chinese version World Health Organization quality of life measure-Abbreviated version (WHOQOL-BREF(HK). Hong Kong: Hong Kong Hospital Authority.

Liao, P. S., Fu, Y. C., \& Yi, C. C. (2005). Perceived quality of life in Taiwan and Hong Kong: An intraculture comparison. Journal of Happiness Studies, 6, 43-67.

Lin, M. R., Yao, K. P., Hwang, J. S., \& Wang, J. D. (1999). Scale descriptor selection for Taiwan-version of questionnaire of World Health Organization quality of life. Chinese Journal of Public Health, 18, 262270 (in Chinese).

Markus, H. R., \& Kitayama, S. (1991). Culture and the self: Implication for cognition, emotions, and motivation. Psychological Review, 98, 224-253.

Markus, H. R., \& Kitayama, S. (1994). A collective fear of the collective: Implication for selves and the theory of selves. Personality and Social Psychology Bulletin, 20, 569-579.

Oishi, S., Diener, E. F., Lucas, R. E., \& Suh, E. M. (1999). Cross-cultural variations in predictors of life satisfaction: Perspectives from needs and values. Personality and Social Psychology Bulletin, 25, 980-990.

Power, M., Harper, A., \& Bullinger, M. (1999). The World Health Organization WHOQOL-100: Tests of the universality of quality of life in 15 different cultural groups worldwide. Health Psychology, 18, 495-505.

Rudmin, F. W. (1994). Cross-cultural psycholinguistic field research: Verbs of ownership and possession. Journal of Cross-Cultural Psychology, 25, 114-132.

Sartorius, N., \& Kuyken, W. (1994). Translation of health status instruments. In J. Orley \& W. Kuyken (Eds.), Quality of life assessment: International perspectives (pp. 3-18). Berlin: Springer.

Shih, F. J. (1996). Concepts related to Chinese patients' perceptions of health, illness and person: Issues of conceptual clarity. Accident and Emergency Nursing, 4, 208-215.

Skevington, S. M., Bradshaw, J., \& Saxena, S. (1999). Selecting national items for the WHOQOL: Conceptual and psychometric considerations. Social Science and Medicine, 48, 473-487.

Skevington, S. M., Lotfy, M., \& O'Connell, K. A. (2004a). The World Health Organization's WHOQOLBREF quality of life assessment: Psychometric properties and results of the international field trial, a Report from the WHOQOL Group. Quality of Life Research, 13, 299-310.

Skevington, S. M., Sartorius, N., \& Amir, M. (2004b). Developing methods for assessing quality of life in different cultural settings. The history of the WHOQOL instruments. Social Psychiatry and Psychiatric Epidemiology, 39, 1-8.

Skevington, S. M., \& Tucker, C. (1999). Designing response scales for cross-cultural use in health care: Data from the development of the UK WHOQOL. British Journal of Medical Psychology, 72, 51-61.

Sprecher, S., \& Toro-Morn, M. (2002). A study of men and women from different sides of earth to determine if men are from Mars and women are from Venus in their beliefs about love and romantic relationships. Sex Roles, 46, 131-147. 
Szabo, S. (1996). The World Health Organization Quality of Life (WHOQOL) assessment instrument. In B. Spiker (Ed.), Quality of life and pharmacoeconomics in clinical trials (pp. 355-362). Philadelphia: Lippincott-Raven.

Szabo, S., Orley, J., \& Saxena, S. (1997). An approach to response scale development for cross-cultural questionnaires. European Psychologist, 2, 270-276.

Taylor, D. M., Simard, L. M., \& Aboud, F. E. (1972). Ethnic identification in Canada: A cross-cultural investigation. Canadian Journal of Behavioral Science, 4, 13-20.

The WHOQOL Group. (1994a). The development of the World Health Organization Quality of Life assessment instrument (the WHOQOL). In J. Orley \& W. Kuyen (Eds.), Quality of life assessment: International perspectives (pp. 41-57). Berlin: Springer.

The WHOQOL Group. (1994b). Development of the WHOQOL: Rationale and current status. International Journal of Mental Health, 23, 24-56.

The WHOQOL Group. (1995). The World Health Organization Quality of Life assessment (WHOQOL): Position paper from the World Health Organization. Social Science and Medicine, 41, 1403-1409.

The WHOQOL Group. (1998a). The World Health Organization Quality of Life assessment (WHOQOL): Development and general psychometric properties. Social Science and Medicine, 46, 1569-1585.

The WHOQOL Group. (1998b). Development of the World Health Organization WHOQOL-BREF quality of life assessment. Psychological Medicine, 28, 551-558.

The WHOQOL-Taiwan Group. (2000). Introduction to the development of the WHOQOL-Taiwan version. Chinese Journal of Public Health, 19, 315-324 (in Chinese).

The WHOQOL-Taiwan Group. (2005). The user's manual of the development of the WHOQOL-100 Taiwan version (2nd ed.). Taipei: The WHOQOL-Taiwan Group (in Chinese).

Triandis, H. C. (1994). Individualism and collectivism: Conceptual clarification and elaboration. In U. Kim, H. C. Triandis, C. Kagitcibasi, S. C. Choi \& G. Yoon (Eds.), Individualism and collectivism: Theory, method, and applications (pp. 41-51). London: Sage.

Tzeng, O. C., \& Everett, A. V. (1985). A cross-cultural perspective of self-related conceptions in adolescence. International Journal of Psychology, 20, 329-348.

Tzeng, O. C., Osgood, C. E., \& May, W. H. (1976). Idealized cultural differences in kinship conceptions. International Journal of Psycholinguistics, 55, 1-77.

Wang, A. (2002). Validation of a self-control rating scale in a Chinese preschool. Journal of Research in Childhood Education, 16, 189-201.

Ware, J. E., Kosinski, M., Gandek, B., Aaronson, N. K., Apolone, G., Bech, P., et al. (1998). The factor structure of the SF-36 health survey in ten countries: Results from the IQOLA project. Journal of Clinical Epidemiology, 51, 1159-1165.

World Health Organization. (1992). WHOQOL focus group moderator training. Geneva: WHO (MNH/PSF/ 92.9).

World Health Organization. (1993). WHOQOL study protocol. Geneva: WHO (MNH/PSF/93.9).

World Health Organization. (1994). WHOQOL protocol for new centers. Geneva: WHO (MNH/PSF/94.4).

World Health Organization (1995a). Field trial WHOQOL-100: Introduction and background. Geneva: WHO (MNH/PSF/95.1.A).

World Health Organization. (1995b). Resources for new WHOQOL centers. Geneva: WHO (MNH/PSF/95.2).

World Health Organization. (1995c). Field trial WHOQOL-100: The 100 questions with response scales. Geneva: WHO (MNH/PSF/95.1.D.Rev.1).

World Health Organization. (1995d). Field trial WHOQOL-100: Scoring the WHOQOL. Geneva: WHO (MNH/PSF/95.1. F).

World Health Organization. (1996). WHOQOL-BREF: Introduction, administration, scoring and generic version of the assessment-Field trial version. Geneva: WHO.

Xia, G., \& Qian, M. (2001). The relationship of parenting style to self-reported mental health among two subcultures of Chinese. Journal of Adolescence, 24, 251-260.

Yao, G., Chung, C. W., Yu, C. F., \& Wang, J. D. (2002). Development and verification of validity and reliability of the WHOQOL-BREF Taiwan version. Journal of the Formosan Medical Association, 101, 342-351.

Yao, G., Lee, P. C., Chen, C. M., \& Wang, J. D. (2007a). Applicability of the WHOQOL-BREF to the general population. Unpublished report. Department of Psychology, National Taiwan University.

Yao, G., Wang, J. D., \& Chung, C. W. (2007b). Cultural adaptation of the WHOQOL questionnaire for Taiwan. Journal of the Formosan Medical Association, 106, 592-597.

Yao, G., \& Wu, C. H. (2005). Factorial invariance study among disease groups using the WHOQOL-BREF Taiwan. Quality of Life Research, 14, 1881-1888.

Zhang, J. B. (1997). Leisure participation frequencies, preferences and perceived barriers among adult Chinese immigrants from three subcultures in New York City: A comparative study. Dissertation Abstracts International Section A: Humanities and Social Sciences. 58(1-A), 288. 